\title{
Mycobiome in the Middle Ear Cavity with and Without Otitis Media with Effusion
}

\author{
Original Investigation

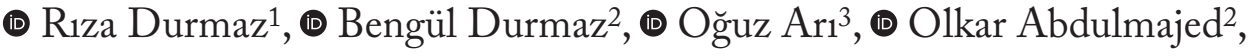

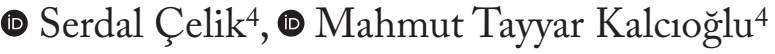 \\ ${ }^{1}$ Department of Clinical Microbiology, Ankara Yildırım Beyazıt University, Faculty of Medicine, Ankara, Turkey \\ ${ }^{2}$ Department of Clinical Microbiology, Yuksek İhtisas University Faculty of Medicine, Ankara, Turkey \\ ${ }^{3}$ Central Research and Application Center, Ankara Yıldırım Beyazıt University, Ankara, Turkey \\ ${ }^{4}$ Department of Otorhinolaryngology, Göztepe Training and Research Hospital, İstanbul Medeniyet University Faculty \\ of Medicine, İstanbul, Turkey
}

\section{Abstract}

\section{ORCID ID of the authors:}

R.D. 0000-0001-6561-778X; B.D. 0000-0002-4519-8730; O.A. 0000-0003-4930-5035; Ol.A. 0000-0002-6420-6185; S.C. 0000-0001-8469-1547; M.T.K. 0000-0002-6803-5467.

Cite this article as: Durmaz $R$, Durmaz $B$ Arı 0, Abdulmajed O, Çelik S, Kalcıoğlu MT. Mycobiome in the Middle Ear Cavity with and Without Otitis Media with Effusion. Turk Arch Otorhinolaryngol 2021; 59(4): 261-70.

Corresponding Author: Riza Durmaz; rdurmaz@ybu.edu.tr

Received Date: 16.10 .2021 Accepted Date: 21.12.2021

Content of this journal is licensed under a Creative Commons Attribution 4.0 International License. Available online at www.turkarchotolaryngol.net

\section{(c) (1) (\$)}

Objective: No data have yet been published revealing the composition and the diversity of fungal communities (mycobiome) in the human middle ear cavity. The presented study investigated the mycobiome in the middle ear cavities of individuals with healthy middle ears and patients with otitis media with effusion.

Methods: A total of 77 middle ear and four adenoid samples were collected from 47 individuals ( 35 children and 12 adults) in Group 1 and from 20 children in Group 2. The mycobiome profile was analyzed with nuclear ribosomal internal transcribed spacer 2 (ITS2) based metabarcoding using an Illumina MiSeq metagenomics kit.

Results: ITS2-based metabarcoding detected 14 different genera and 17 different species with a mean relative abundance of $\geq 1 \%$ in the samples analyzed. Mycobiome profile was similar between the adenoid tissue and the middle ear cavity, between Groups 1 and Group 2, and between children and adults. Fusarium, Stemphylium, Candida, and Cladosporium were the most abundant genera detected in all samples. The mean relative abundances of the genera Candida and Fusarium were remarkably higher in Group 2 compared to Group 1.

Conclusion: The species Candida glaebosa, Candida cretensis, Aspergillus ruber, Penicillium desertorum, and Rhizopus arrbizus were significantly more abundant in patients with otitis media with effusion $(\mathrm{OME})$, raising the possibility that they affect the pathogenesis of OME.

Keywords: Mycobiome, fungus, middle ear, adenoid, otitis media with effusion, high-throughput sequencing, metabarcoding 


\section{Introduction}

Mycobiome, the fungal community in and on an organism, makes up less than $1 \%$ of the human microbiome (1).Although the diversity and relative abundance of mycobiome are lower than those of the bacteriome of microbiota, certain body sites such as the oral cavity, respiratory tract, gastrointestinal tract, skin, and vagina host several fungal genera in addition to bacteriome $(2,3)$. Regarding the mycobiome of the oral cavity and the respiratory tract, which interacts with the middle ear cavity, culture-depended and molecular techniques revealed diverse genera such as Candida, Cladosporium, Saccharomyces, Penicillium and Aspergillus, in these anatomic sites (4-6).

Fungi on and in humans play an important role in health and disease development alike by modifying basic physiology and maintaining microbial community profiles (7). Due to the small number of studies, however, we have limited knowledge on the interaction between mycobiome and host. Accurate identification of the fungi causing invasive and noninvasive infection is of critical importance. Advances in molecular technologies offer far-reaching insight into the human mycobiome in health and disease (5). High-throughput sequencing (HTS) approaches used to characterize the microbiome commonly rely on the amplification and sequencing of relatively short DNA regions. For fungi, the internal transcribed spacer 1 (ITS1) located between ribosomal subunits $18 \mathrm{~S}$ and $5.8 \mathrm{~S}$ rRNA regions and ITS2 located between $5.8 \mathrm{~S}$ and $28 \mathrm{~S}$ regions in the ribosomal RNA (rRNA) operon have been widely used as targets for analyzing the mycobiome composition in diverse ecosystems $(3,6,8)$. Recent studies indicate a preference for targeting the ITS2 region as it includes more universal primer sites and leads to less taxonomic bias $(3,8)$.

Otitis media with effusion (OME) are a common problem in children, although its pathogenesis remains incompletely understood (9). According to general opinion, immunological, anatomic, genetic, microbial, and environmental factors contribute to the development of this condition $(10,11)$. Using polymerase chain reaction (PCR) and HTS-based approaches revealed a diverse bacteriome in the middle ear cavities of individuals with and without otitis media with effusion (12-14). There are no published data indicating fungal compositions in the middle ear cavity, which is connected to the respiratory tract. There are also no data on whether mycobiome profile changes in the presence of OME.

Previously we reported, for this same set of samples, that both healthy middle ears and ear with OME have diverse bacteriomes and viromes $(12,14,15)$. Here, we hypothesize that the middle ear hosts a mycobiome and the composition of the mycobiome differ according to the health condition of the middle ear. To pursue this hypothesis, 77 middle ear and four adenoid tissue samples collected from 47 healthy individuals (12 adults and 35 children) undergoing cochlear implant surgery and 20 children with OME were analyzed using High-throughput sequencing of the ITS2.

\section{Methods}

A total of 81 samples, which were previously collected for bacteriome analyses in our previous studies and stored at -32 ${ }^{\circ} \mathrm{C}$, were used in this study. Briefly, of the 77 middle ear fluid samples, 57 were taken from the 47 individuals $(20$ samples were obtained from both right and left ears) with no middle ear disease and who had undergone cochlear implant surgery. Thirty-five of the 47 patients were in the child (younger than 4 years) and 12 in the adult ( $\geq 18$ years) age group (Group 1) (12). Twenty middle ear samples were obtained from 20 children (aged 1.5 to 9 years) who had bilateral or unilateral persistent chronic OME for six months or longer (Group 2). Additionally, four adenoid tissue samples of the participants in Group 2 were analyzed (14).

The research protocols for Groups 1 and 2 were approved by the İstanbul Medeniyet University Clinical Research Ethics Committee with the protocol numbers 2018/0313 and 2011/103, respectively. The protocols were applied in accordance with the Declaration of Helsinki. Informed consent was obtained from all adult patients and from the parents of the children included in the study.

DNA isolation was done using the GeneMATRIX Plant \& Fungi DNA Purification kit (EURx Ltd. 80-297, Gdansk, Poland) following the manufacturer's instructions (https:// eurx.com.pl/docs/manuals/en/e3595.pdf). To eliminate environmental contamination, we followed strict sterility conditions. The Qubit 3.0 Fluorometer (Thermo Fisher Sci, Warrington, England) was used for the quantitation of DNA, and $12.5 \mathrm{ng}$ DNA of each sample was used to amplify about 390 bases of the ITS2 region.

Amplification of the ITS2 region was performed using the MiSeq adaptor (underlined) containing primers ITS3 (TCGTCGGCAGCGTCAGATGTGTA TAAGAGACAGGCATCGATGAAGAACGCAGC)and ITS4 (GTCTCGTGGGCTCGGAGATGTGTATAAG AGACAGTCCTCCGCTTATTGATATGC) following the Illumina fungal metagenomic-sequencing demonstrated protocol (https://support.illumina.com/downloads/fungal -metagenomic-sequencing-demonstrated-protocol1000000064940.html).

PCR products were purified using the Agencourt AMPureXP kit (Beckman Coulter Inc., Brea, CA, USA). DNA concentrations were measured in a Qubit 3.0 Fluorometer device using the Qubit ${ }^{\mathrm{TM}}$ dsDNA HS Assay Kit (http://tools.thermofisher. com/content/sfs/manuals/ qubit_3_fluorometer_man.pdf). Following Illumina's ITS metagenomics demonstrated protocol (https://support. 
illumina.com/downloads/fungal-metagenomic-sequencingdemonstrated-protocol-1000000064940. html), the PCR products were barcoded using the Nextera index primers 1 and 2 (Illumina, San Diego, CA, USA) and the 2X KAPA HotStart PCR mixture (Kapa Biosystems, Wilmington, MA, USA). AMPure XP beads were used to clean up the index PCR products. Barcoded samples were quantified using the Qubit ${ }^{\mathrm{TM}}$ dsDNA HS Assay Kit (Thermo Fisher Sci, Warrington, UK) and equally multiplexed into a single library. Sequencing of paired end libraries was performed on an Illumina MiSeq Sequencing platform (Illumina, San Diego, CA, USA) employing the MiSeq Reagent Kit v3 $(2 \times 300$ cycles) as recommended by the manufacturer (https:// sapac.support.illumina.com/content/dam/illumina-support/ documents/documentation/chemistry_documentation/ metagenomic/fungal-metagenomic-demonstratedprotocol-1000000064940-01.pdf). All samples were sequenced on the same MiSeq run.

Raw sequencing data were demultiplexed and adaptor was trimmed using the MiSeq system. The paired end raw reads obtained were controlled using specific filtering conditions according to the Quantitative Analysis in Microbial Ecology (QIIME2 version 2019.10.0) (16). The raw reads were imported as a QIIME2 artifact. Then, the data were cleaned using DADA2. QIIME DADA2 denoise-paired option was used with specific options based on sequencing statistics to filter out errors, chimeras, low-quality reads, and quality trimming (17). The amplicon sequence variants (ASVs) produced by DADA2 were mapped to Unite (v8.2) database. Fungal taxa were created based on a clustering at the $97 \%$ threshold level (18).

\section{Statistical Analysis}

Alpha diversity metrics were calculated using the microbiome $\mathrm{R}$ package (19). For statistical analysis, $\mathrm{R}$ packages phyloseq (v1.34), vegan (v2.5) and microbiome (v1.13) were used (20). $\mathrm{P}$ values of alpha diversity metrics were calculated with the Kruskal-Wallis test. Spearman's correlation coefficient was used to evaluate the degree of correlation between the microbiomes of the patients with and without OME (https://statistics.laerd.com/statistical-guides/spearmansrank-order-correlation-statistical-guide-2.php). The beta diversity of the microbial community profile between samples of adenoid tissue and the middle ear cavity with and without OME was tested by Permutational Multivariate Analysis of Variance (PERMANOVA) using the Bray-Curtis dissimilarity, the unweighted and weighted UniFrac distances, the Jaccard parameters (https://www.rdocumentation.org/ packages/vegan/versions/2.4-2/topics/adonis). Analysis of similarities (ANOSIM) based on UniFrac distances were used to evaluate the dissimilarity in microbiome composition within and between study groups (https://sites. google.com/site/mb3gustame/hypothesis-tests/anosim).
We used the linear discriminant analysis (LDA) effect size (LEfSe) to define the potential biomarkers with differences in abundance between the samples (21). Relative abundances were calculated by normalizing the ASVs table of raw counts and statistical analysis was performed on the taxa with $\geq 1 \%$ total relative abundance across samples. A non-parametric Mann-Whitney test was applied to determine whether there were statistically significant differences in the relative abundances of taxa detected between patients with and without OME (22).

\section{Results}

ITS2 metabarcoding analysis of the 81 samples yielded 1074 970 mapped sequence reads. Two samples (ID numbers S94 and S574) collected from Group 1 were excluded because of very low number of reads. A total of 14 different fungal genera and 17 species with a mean relative abundance of $\geq 1 \%$ were found from the 79 samples analyzed (Table 1). Alfa diversity analysis showed no statistically significant difference in the number of taxa, community richness and the diversity between the mycobiome of the adenoid samples and the middle ear cavity samples of the patients in Group 2, between mycobiome of Groups 1 and 2, and between the mycobiome of children and adults in Group 1 (Supplementary Figure 1). Spearman's correlation coefficient revealed slightly negative correlation between Group 1 and Group $2(-0.06256937)$ and between adenoid samples and Group $2(-0.1025119)$

The analysis of samples collected from the patients with OME (Group 2) revealed that the relative abundance of the genera detected within each adenoid and middle ear sample showed substantial variation. For instance, the abundance of the predominant genus Fusarium-which was found in all samples-varied between $14.9 \%$ and $42.4 \%$ in the adenoid, between $8.2 \%$ and $62.5 \%$ in the middle ear samples. Similarly, the second most common genus Stemphylium was detected in all samples and its relative abundance varied from $16.8 \%$ to $40.8 \%$ in the adenoid and from $4.5 \%$ to $48.4 \%$ in the middle ear samples. The relative abundance of the third most common genus Candida found that all samples were between $1.1 \%$ and $9.4 \%$ in the adenoid tissue and between $1.8 \%$ and $64.8 \%$ in the middle ear samples (Figure 1).

According to the mean relative abundance of the 14 genera found in Group 2, Fusarium had the highest mean relative abundance accounting $30.4 \%$ of the total abundance followed by Candida (15.9\%) Stemphylium (15.7\%), and others with a mean relative abundance between $0.6 \%$ and 6.6\%. The prevalence of the 14 genera varied from $95 \%$ to $100 \%$. In the adenoid samples, Fusarium, Stemphylium and Candida were the three dominant genera accounting 59.4\% of the total abundance. According to the Mann-Whitney test results, there was only statistically significant differences 


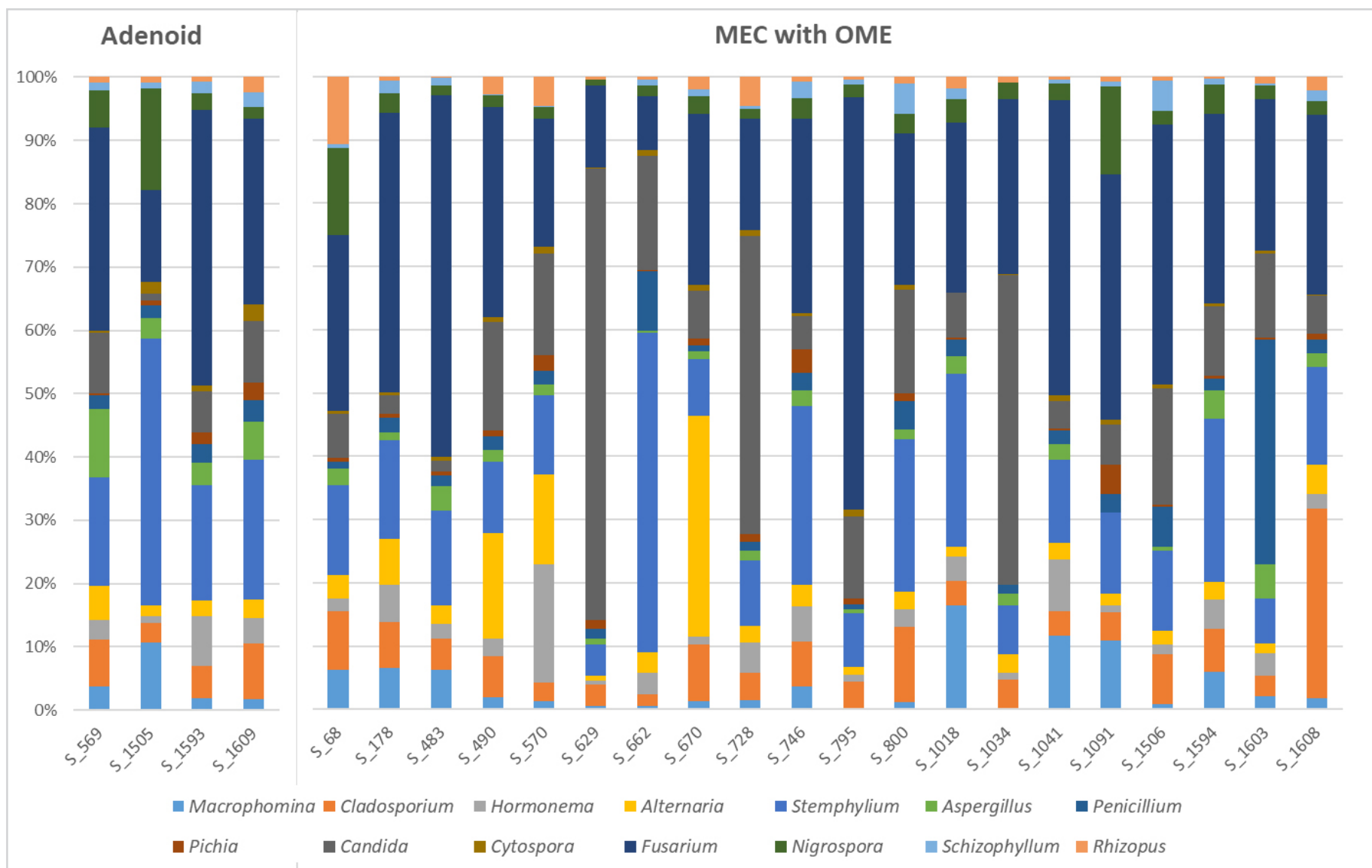

Figure 1. The relative abundance of fungal genera detected from the samples of adenoid and the middle ear cavities (MEC) with OME. Fusarium, Stemphylium and Candida were the most abundant genera found in all adenoid and middle ear samples. Each had different relative abundance, $8.2 \%$ to $62.5 \%$ for Fusarium, $4.5 \%$ to $48.4 \%$ for Stemphylium and $1.1 \%$ to $64.8 \%$ for Candida.

OME: Otitis media with effusion

in mean relative abundance of the genus Aspergillus (Table 1). ANOSIM analysis confirmed that there were no significant dissimilarities in the microbiome compositions within and between the adenoid tissues and the middle ear cavity samples of the patients with OME ( $\mathrm{p}=0.65)$.

ITS2 metabarcoding analysis of the 45 middle ear samples collected from the patients without OME (Group 1) showed that Fusarium, Stemphylium, Cladosporium and Candida were the most commonly detected genera in all samples from adults and children, however their relative abundance within the samples showed variations. For instance, the relative abundance of Fusarium was between $14.0 \%$ and $34.5 \%$ in adults, and between $8.3 \%$ and $46.0 \%$ in children (Figure 2).

Regarding the mean relative abundance of the genera detected in Group 1, Fusarium was the most abundant genus with a mean relative abundance of $24.9 \%$ in children and $26.1 \%$ in adults, followed by Stemphylium (18.6\% and $19.0 \%$, respectively), Candida (11.7\% and 8.8\%, respectively) and Cladosporium (9.3\% and 9.5\%, respectively). Other genera detected in both children and adults had mean relative abundance between $0.62 \%$ and $7.6 \%$. There was no significant difference between children and adults in terms of the mean relative abundance of each. The prevalences of these genera were more than $98 \%$. Like the distribution of genera, the mean relative abundance of the fungal species identified in adults and children did not show a statistically significant difference, except for the significant abundance of Penicillium atrosanguineum in the children.

ANOSIM analysis revealed the presence of significant dissimilarity in the microbiome composition within and between Groups 1 and $2(\mathrm{p}=0.049)$. Aspergillus and Rhizopus were significantly more abundant in Group 2 than in Group 1 ( $\mathrm{p}=0.000)$. However, Cladosporium, Pichia, Cytospora, Nigrospora and Schizophyllum were more abundant in Group 1 ( $\mathrm{p}$-values $\leq 0.001)$. Although there were no significant differences, the mean relative abundances of the genera Candida (15.9\% vs $11.1 \%$ ) and Fusarium (30.4\% vs $25.1 \%$ ) were higher in Group 2 vs Group 1 (Figure 3). The species Candida glaebosa, Candida cretensis, Aspergillus ruber, Penicillium desertorum, and Rbizopus arrbizus were significantly more abundant in patients with OME than in patients with a healthy middle ear (Table 1). Significant difference between Group 1 and Group 2 in the species 
Table 1. Mean relative abundance of the genera and the species detected in the study groups

Mean relative abundance (\%)

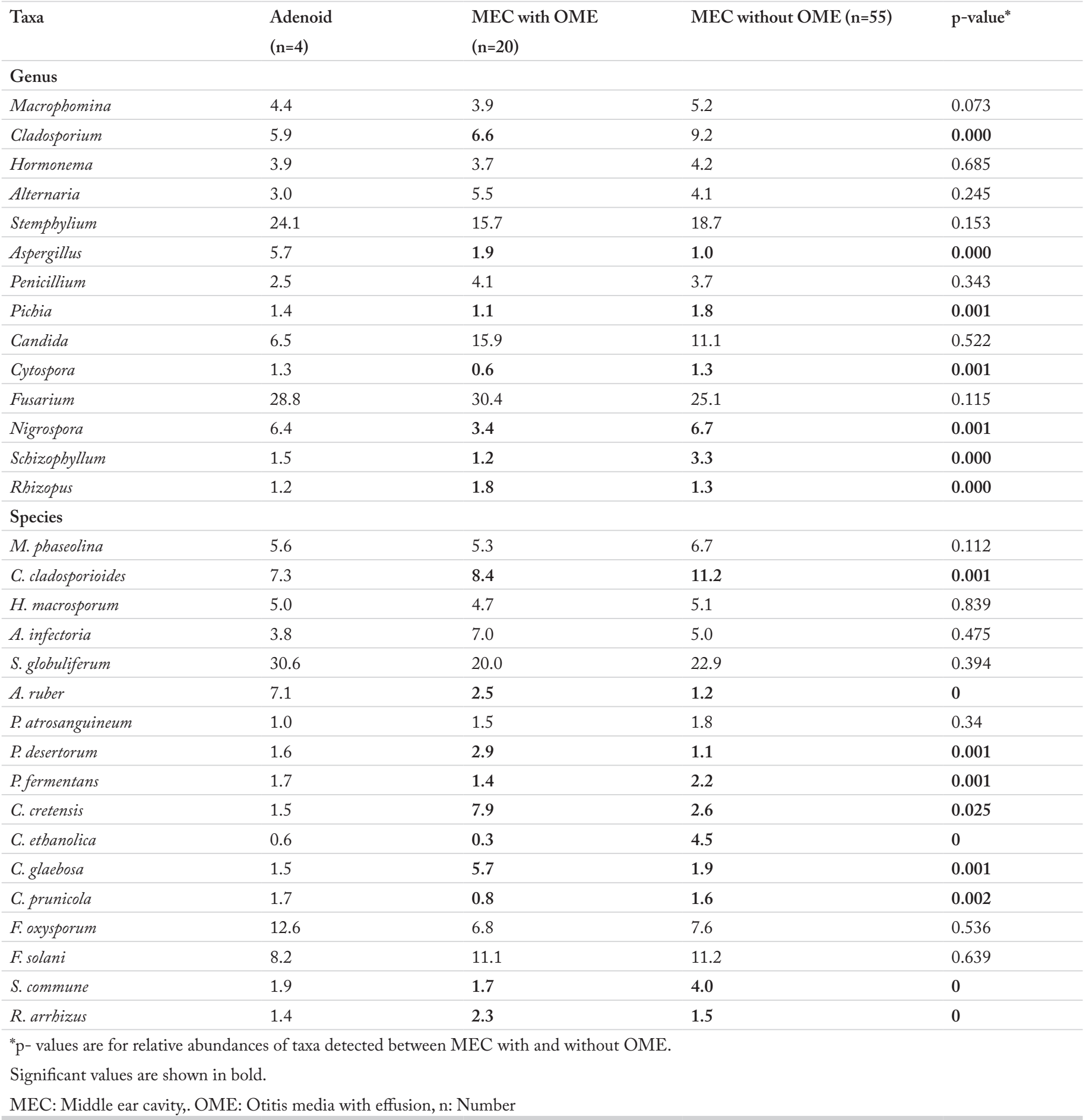

compositions was confirmed with Bray-Curtis dissimilarity $(p=0.001)$, the unweighted $(p=0.011)$ and weighted $(p=0.003)$ UniFrac distances, and the Jaccard analysis ( $\mathrm{p}=0.003)$. LEfSe analysis revealed that these species except $A$. ruber, could be used as biomarker for the diagnosis of otitis media with effusion (Table 2).

\section{Discussion}

Analysis of the mycobiome by using HTS technologies has highlighted the presence of diverse cultivable and non-cultivable fungi in all areas of the human body explored, including areas thought to be microbiologically barren $(1,23)$. PCR approaches 


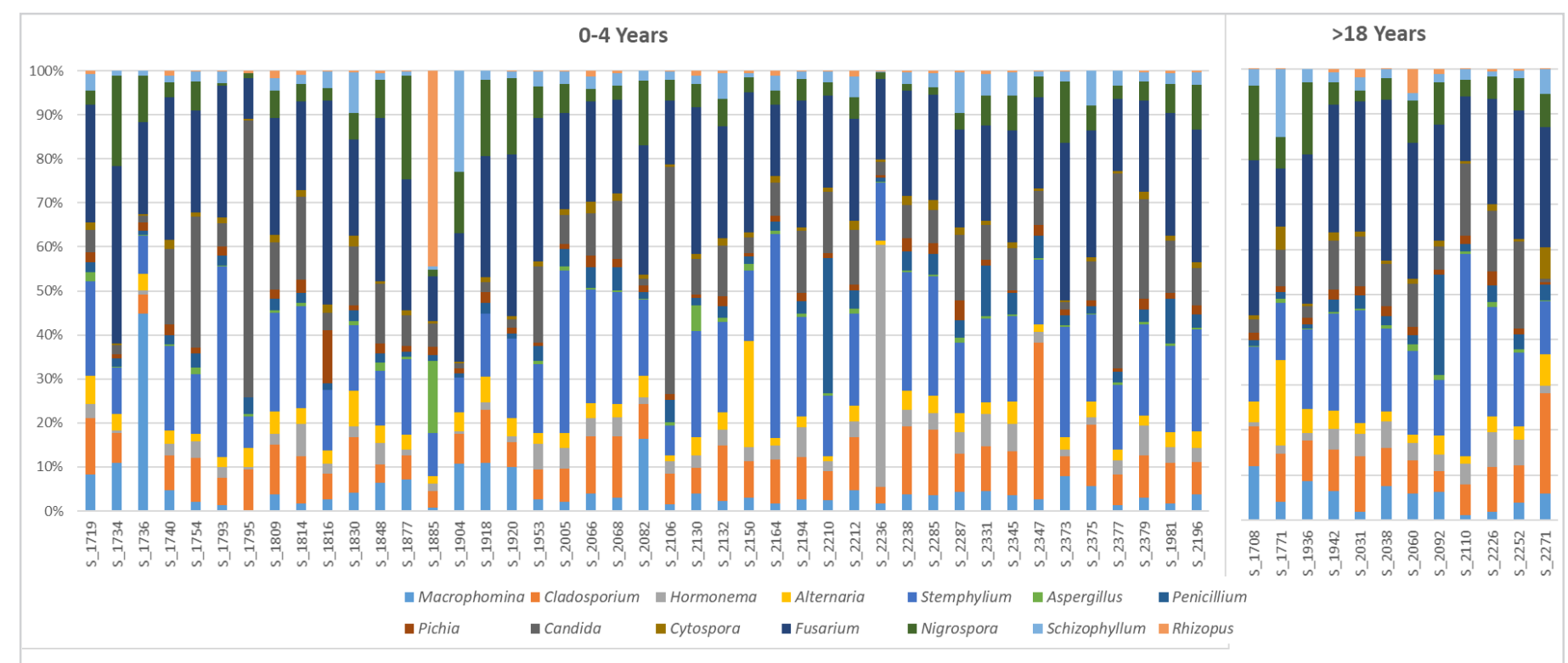

Figure 2. The relative abundance of fungal genera detected from the middle ear cavities without OME. Fusarium, Stemphylium, Cladosporium, and Candida were the most commonly detected genera in all samples from both adults and children. Their relative abundance showed big differences within each sample. The relative abundance varied between $8.3 \%$ and $46.0 \%$ for Fusarium, $6.4 \%$ and $44.8 \%$ for Stemphylium, and $0.7 \%$ and $56.4 \%$ for Candida. Similar variation was observed for the remaining rare genera.

OME: Otitis media with effusion

Table 2. Potential biomarkers defined using LEfSe analysis for diagnosis of the otitis media with effusion

\begin{tabular}{llll} 
Marker Species & LDA score & p-value & p-value adjusted \\
\hline Candida cretensis & 4.753616259 & 0.001852798 & 0.001852798 \\
\hline Candida glaebosa & 4.645068076 & 0.000139425 & 0.000139425 \\
\hline Penicillium desertorum & 4.284159638 & 0.005567622 & 0.005567622 \\
\hline Trichosporon asabii & 4.209985054 & 0.001420361 & 0.001420361 \\
\hline Rhizopus arrbizus & 4.202264894 & 0.000131602 & 0.000131602 \\
\hline
\end{tabular}

LEfSe: Linear discriminant analysis effect size, LDA: Linear discriminant analysis

showed fungal DNA in the middle ear cavities of the patients; however, these studies did not give any information about fungal genera and or species $(24,25)$. Our study is the first study that used high-throughput sequencing to evaluate the mycobiome profiles in the middle ear with and without OME.

The presented study showed diverse fungal DNA in the middle ear cavity. With regards to the mycobiome in the middle ear samples of patients with OME (Group 2), the presented study is the first report showing at least 14 different fungal genera with a relative abundance of $\geq 1 \%$ in the middle ear cavity. In contrast to a previous study reporting high diverse bacteriome, we found a lower number of fungal genera in the same samples (14). These data agreed with the idea indicating that the diversity of the fungal taxa per sample was lower than those of bacteria in the microbiota (2).
The genera detected in the middle ear cavities of the patients in Group 2 were previously reported in samples of human oral mycobiome $(1,4)$. As in our results, Candida and Cladosporium were reported as the most prevalent genera found in $75 \%$ and $65 \%$ of oral samples of the 20 healthy participants in a previous study. In that study, Fusarium, Aureobasidium, Saccharomycetales, Aspergillus and Cryptococcus were also found with a remarkable prevalence varying between $20 \%$ and $50 \%$ (4). We found that the prevalence of genera varied from $90 \%$ to $100 \%$. Besides profile similarity, the relative abundance, richness, and prevalence of the genera detected within each individual in Group 2 showed remarkable variation. The reason for these differences may stem from the personal characteristics, lifestyles, and life histories of the participants $(2,5)$.

To conclude whether the mycobiome in the middle ear originates from adenoid or whether there is a core mycobiome in the middle ear cavity, we analyzed both adenoid and 


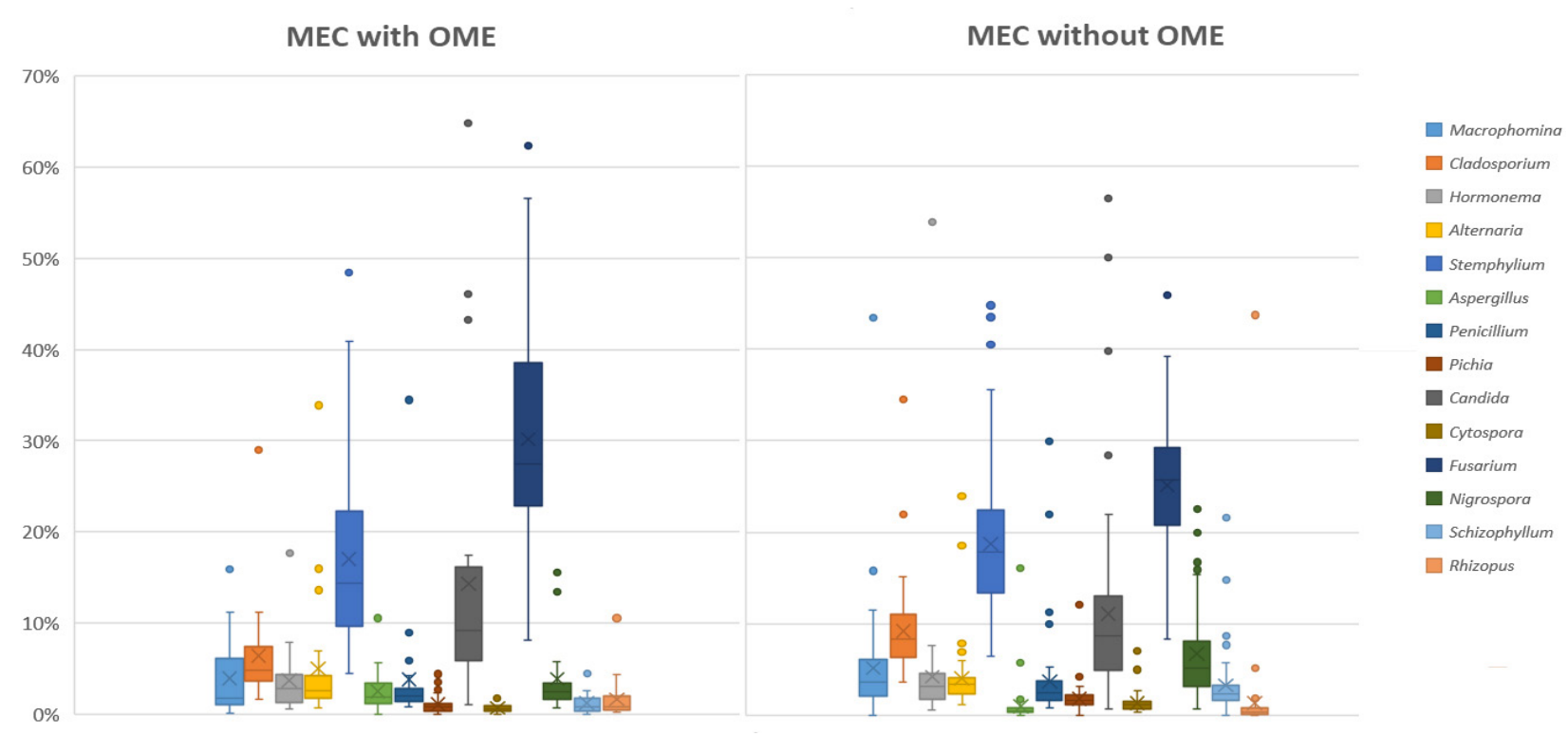

Figure 3. Box and whisker plot of relative abundance of fungal genera detected in the middle ear cavity with and without OME. Fourteen common genera were found from all samples. The genera Aspergillus and Rhizopus were significantly more abundant in the middle ear cavities with OME (p-values 0.000 and 0.000, respectively), whereas the genera Cladosporium, Pichia, Cytospora, Nigrospora and Schizophyllum were more abundant in Group 1 ( $\mathrm{p}$-values $\leq 0.001$ ).

OME: Otitis media with effusion

middle ear samples of the four patients. There was an overlap between adenoid and middle ear mycobiome. Although the number of samples was too limited for us to arrive at a robust conclusion, this finding supports the idea that the microbiome of the middle ear come from the oropharynx and/or nasopharynx (13). Cui et al. (26) revealed overlapping of the mycobiome communities between bronchoalveolar lavages and oral washes, suggesting that some fungi detected in lung originated from the mouth. Although no a study that compared the oral and middle-ear cavities, the results of Cui et al. (26) lend weight to a mycobiome transfer between adenoid tissue and the middle ear cavity.

To analyze whether the middle ear without OME had mycobiome and whether mycobiome varied by age, we analyzed 55 middle ear samples, 12 were from adults and 35 were from children. Our study did not show any dissimilarity in the mycobiome profile between children younger than 4 years and adults ( $\geq 18$ years). There were also no significant differences in relative abundance, and prevalence of the detected genera and species, except for significantly more abundance of $P$ atrosanguineum in the children. From these results we can speculate that the mycobiome in the middle ear is established during the first years of life and stabilized in adults. In parallel to these results, in our previous study conducted on the same samples, we found similar bacteriome composition between adults and children (12).
ANOSIM and PERMANOVA analyses revealed significant dissimilarities in the observed fungal taxa between the middle ear with and without OME. Fusarium (F. solani and F. oxysporum) and Candida (C. cretensis, C. glaebosa and C. ethanolica) were the two genera showing remarkably more abundance in the middle ears with OME. These opportunistic fungi can invade different organs and cause serious diseases. The genus Candida is the most common fungus resulting in different invasive infections (27). Recently, human infections caused by $F$. oxysporum and F. solani (Neocosmospora solani) have increased and become the second most common mold infections after aspergillosis (28). Although their mean relative abundance was too low (less than 2\%) Aspergillus (A. ruber) and Rbizopus (R. arrbizus) were significantly more abundant in Group 2 than in Group 1 (p-values, 0.000). A recent meta-analysis indicated the predominance of Rbizopus spp. in patients with rhino-orbital-cerebral mucormycosis (29). In concordance with the literature, one can speculate that these opportunistic taxa contribute to the development of effusion in the middle ear cavity.

The detection of Cladosporium, Pichia, Nigrospora, Cytospora and Schizophyllum with significantly lower abundances in the middle ear with OME than in the middle ear without $\mathrm{OME}$ and presence of these genera also in adenoid samples indicate their portal entry into the middle ear via Eustachian tube. From these data, one can speculate that these genera are present in the middle ear as transient mycobiome members 
and they may not be involved in the development of effusion. However, Stemphylium (S. globuliferum) was the second most abundant genus in the middle ear with and without OME ( $15.7 \%$ vs $18.7 \%)$, respectively. Since this genus is mainly known as a ubiquitous environmental saprobic fungus, it can be evaluated as a mere colonizer in middle ear cavity (30). As Stemphylium was found in oral mycobiome, the genus in middle ear cavity stems from the oral cavity (4).

This study has some limitations that should be noted. While the high-throughput sequencing revealed diverse mycobiome in the healthy middle ear cavities with and without OME, based on these results, we cannot say that the presence of fungal DNA is evidence for viable fungal cells. Further studies using culture-based approaches are needed to clarify whether this fungal DNA detected in the middle ear represents living fungi and to confirm the clinical relevance for the presence of a diverse fungal population in the middle ear cavity. Another limitation of our study is the low number of the adenoid samples.

\section{Conclusion}

This study conducted on many samples to target the middleear mycobiome provided some new data. First, we report evidence for the presence of diverse fungi in middle ear cavities with and without OME. Second, the presence of similar mycobiomes across the middle ear cavity and adenoid sample of the same participant supports the idea that the mycobiome in the middle ear stems from adenoid tissue. Third, the microbiome profile in the middle ear cavities of children and adults had similar relative abundance, richness, and prevalence. Four, higher abundance of the species $C$. glaebosa, C. cretensis, A. ruber, $P$. desertorum and $R$. arrbizus in patients with OME suggests that these taxa contribute to inflammation and development of effusion. Based on LEfSe analysis, these species except $A$. rubrum, can be potential biomarkers for microbiologic diagnosis of otitis media with effusion. We believe that these results will point to a novel perspective to evaluate the pathogenesis of the effusion.

Acknowledgment: Authors thanks to Ahmet Varı̧̧ and Samet Ece at Diagen Biotechnological Systems Inc. Ankara, Turkey for the bioinformatics analysis of sequencing data.

Ethics Committee Approval: The research protocols were approved by the İstanbul Medeniyet University Clinical Research Ethics Committee with the protocol numbers 2018/0313 and 2011/103, respectively.

Informed Consent: Informed consent was obtained from all adult patients and from the parents of the children included in the study.

Peer-review: Externally peer-reviewed.

\section{Authorship Contributions}

Investigation: R.D., Conception: B.D., Methodology: O.A., O1.A., S.C., M.T.K., Data Curation: R.D., S.C., MT.K., Analysis of Data: R.D., O.A., Writing: R.D., Ol.A., Editing Manuscript: B.D., M.T.K., Review: B.D., M.T.K.

Conflict of interest: All authors declare no conflict of interest.

Financial Disclosure: This study was supported by Yüksek İhtisas University Research Foundation with a project number of 2020-01.005.

\section{Main Points}

- This is the first study that used high-throughput sequencing to evaluate the mycobiome profiles in middle ears with and without OME.

- We report evidence for the presence of diverse fungi in middle ear cavities with and without OME.

- We found similar mycobiomes across the middle ear cavity and adenoid samples of the same participant.

- The microbiome profile in the middle ear cavities of children and adults had similar relative abundance, richness and prevalence.

- Higher abundance of the species C. glaebosa, C. cretensis, A. ruber, $P$. desertorum and $R$. arrbizus in the patients with OME suggests that these taxa might contribute to inflammation and development of effusion.

- These results will point to a novel perspective to evaluate the pathogenesis of the effusion.

\section{References}

1. Seed PC. The human mycobiome. Cold Spring Harb Perspect Med 2014; 5: a019810. [Crossref]

2. Tiew PY, Mac Aogain M, Ali NABM, Thng KX, Goh K, Lau KJX, et al. The mycobiome in health and disease: emerging concepts, methodologies, and challenges. Mycopathologia 2020; 185: 207231. [Crossref]

3. Nguyen LD, Viscogliosi E, Delhaes L. The lung mycobiome: an emerging field of the human respiratory microbiome. Front Microbiol 2015; 6: 89. [Crossref]

4. Ghannoum MA, Jurevic RJ, Mukherjee PK, Cui F, Sikaroodi M, Naqvi A, et al. Characterization of the oral fungal microbiome (mycobiome) in healthy individuals. PLoS Pathog 2010; 6: e1000713. [Crossref]

5. Cui L, Morris A, Ghedin E. The human mycobiome in health and disease. Genome Med 2013; 5: 63. [Crossref]

6. Ali NABM, Mac Aogáin M, Morales RF, Tiew PY, Chotirmall SH. Optimisation and benchmarking of targeted amplicon sequencing for mycobiome analysis of respiratory specimens. Int J Mol Sci 2019; 20: 4991. [Crossref] 
7. Shen X, Yao YF, Li JY, Li Y. [Human mycobiome and diseases.] Hua Xi Kou Qiang Yi Xue Za Zhi 2019; 37: 314-9. [Crossref]

8. Op De Beeck M, Lievens B, Busschaert P, Declerck S, Vangronsveld J, Colpaert JV. Comparison and validation of some ITS primer pairs useful for fungal metabarcoding studies. PLoS One 2014; 9: e97629. [Crossref]

9. Ungkanont K, Boonyabut P, Komoltri C, Tanphaichitr A, Vathanophas V. Surveillance of otitis media with effusion in Thai children with cleft palate: cumulative incidence and outcome of the management. Cleft Palate Craniofac J 2018; 55: 590-5. [Crossref]

10. Butler CC, Williams RG. The etiology, pathophysiology, and management of otitis media with effusion. Curr Infect Dis Rep 2003; 5: 205-12. [Crossref]

11. Buzatto GP, Tamashiro E, Proenca-Modena JL, Saturno TH, Prates MC, Gagliardi TB, et al. The pathogens profile in children with otitis media with effusion and adenoid hypertrophy. PLoS One 2017; 12: e0171049. [Crossref]

12. Kalcioglu MT, Durmaz R, Ari O, Celik S, Karabudak S. Microbiological investigation of samples collected from healthy middle ears during cochlear implant surgery. Diagn Microbiol Infect Dis 2021; 100: 115390. [Crossref]

13. Minami SB, Mutai H, Suzuki T, Horii A, Oishi N, Wasano K, et al. Microbiomes of the normal middle ear and ears with chronic otitis media. Laryngoscope 2017; 127: E371-7. [Crossref]

14. Ari O, Karabudak S, Kalcioglu MT, Gunduz AY, Durmaz R. The bacteriome of otitis media with effusion: does it originate from the adenoid? Int J Pediatr Otorhinolaryngol 2019; 126: 109624. [Crossref]

15. Durmaz B, Abdulmajed O, Durmaz R, Koroglu M, Ar1 O, Celik $\mathrm{S}$, et al. Respiratory viruses in healthy middle ear and middle ear with otitis media with effusion. J Med Virol 2021; 93: 6140-7. [Crossref]

16. Bolyen E, Rideout JR, Dillon MR, Bokulich NA, Abnet CC, A1Ghalith GA, et al. Reproducible, interactive, scalable and extensible microbiome data science using QIIME 2. Nat Biotechnol 2019; 37: 852-7. [Crossref]

17. Callahan BJ, McMurdie PJ, Rosen MJ, Han AW, Johnson AJ, Holmes SP. DADA2: high-resolution sample inference from Illumina amplicon data. Nat Methods 2016; 13: 581-3. [Crossref]

18. Nilsson RH, Larsson KH, Taylor AFS, Bengtsson-Palme J, Jeppesen TS, Dmitry Schigel D, et al. The UNITE database for molecular identification of fungi: handling dark taxa and parallel taxonomic classifications. Nucleic Acids Res. 2019; 47(Database issue): D259-D264. [Crossref]
19. Lahti L, Shetty S Blake T, Salojarvi J. Tools for microbiome analysis in R. Microbiome package version. https://microbiome. github.io/tutorials/. Accessed December 4, 2021. [Crossref]

20. McMurdie PJ, Holmes S. Phyloseq: an R package for reproducible interactive analysis and graphics of microbiome census data. PLoS One 2013; 8: e61217. [Crossref]

21. Segata N, Izard J, Waldron L, Gevers D, Miropolsky L, Garrett WS, et al. Metagenomic biomarker discovery and explanation. Genome Biol 2011; 12: R60. [Crossref]

22. Vickers AJ. Parametric versus non-parametric statistics in the analysis of randomized trials with non-normally distributed data. BMC Med Res Methodol 2005; 5: 35. [Crossref]

23. Pisa D, Alonso R, Carrasco L. Parkinson's disease: a comprehensive analysis of fungi and bacteria in brain tissue. Int J Biol Sci 2020; 16: 1135-52. [Crossref]

24. Jalali MM, Rezaie S, Kousha A, Saadat F, Banan R. Detection of fungal DNA in the middle ear effusion of patients suffering from otitis media with effusion. Iranian J Publ Health 2008; 37: 109-13. [Crossref]

25. Kim EJ, Catten MD, Lalwani AK. Detection of fungal DNA in effusion associated with acute and serous otitis media. Laryngoscope 2002; 112:2037-41. [Crossref]

26. Cui L, Lucht L, Tipton L, Rogers MB, Fitch A, Kessinger C, et al. Topographic diversity of the respiratory tract mycobiome and alteration in HIV and lung disease. Am J Respir Crit Care Med 2015; 191: 932-42. [Crossref]

27. Aslan N, Yildizdas D, Alabaz D, Horoz OO, Yontem A, Kocabas E. Invasive candida infections in a pediatric intensive care unit in Turkey: evaluation of an 11-year period. J Pediatr Intensive Care 2020; 9: 21-6. [Crossref]

28. Castillo-Castañeda A, Cañas-Duarte SJ, Guevara-Suarez M, Guarro J, Restrepo S, Celis Ramírez AM. Transcriptional response of Fusarium oxysporum and Neocosmospora solani challenged with amphotericin B or posaconazole. Microbiology (Reading) 2020; 166: 936-46. [Crossref]

29. Jeong W, Keighley C, Wolfe R, Lee WL, Slavin MA, Kong DCM, et al. The epidemiology and clinical manifestations of mucormycosis: a systematic review and meta-analysis of case reports. Clin Microbiol Infect 2019; 25: 26-34. [Crossref]

30. Hwang JY, Park SC, Byun WS, Oh DC, Lee SK, Oh KB, et al. Bioactive bianthraquinones and meroterpenoids from a marinederived Stemphylium sp. Fungus. Mar Drugs 2020; 18: 436. [Crossref] 


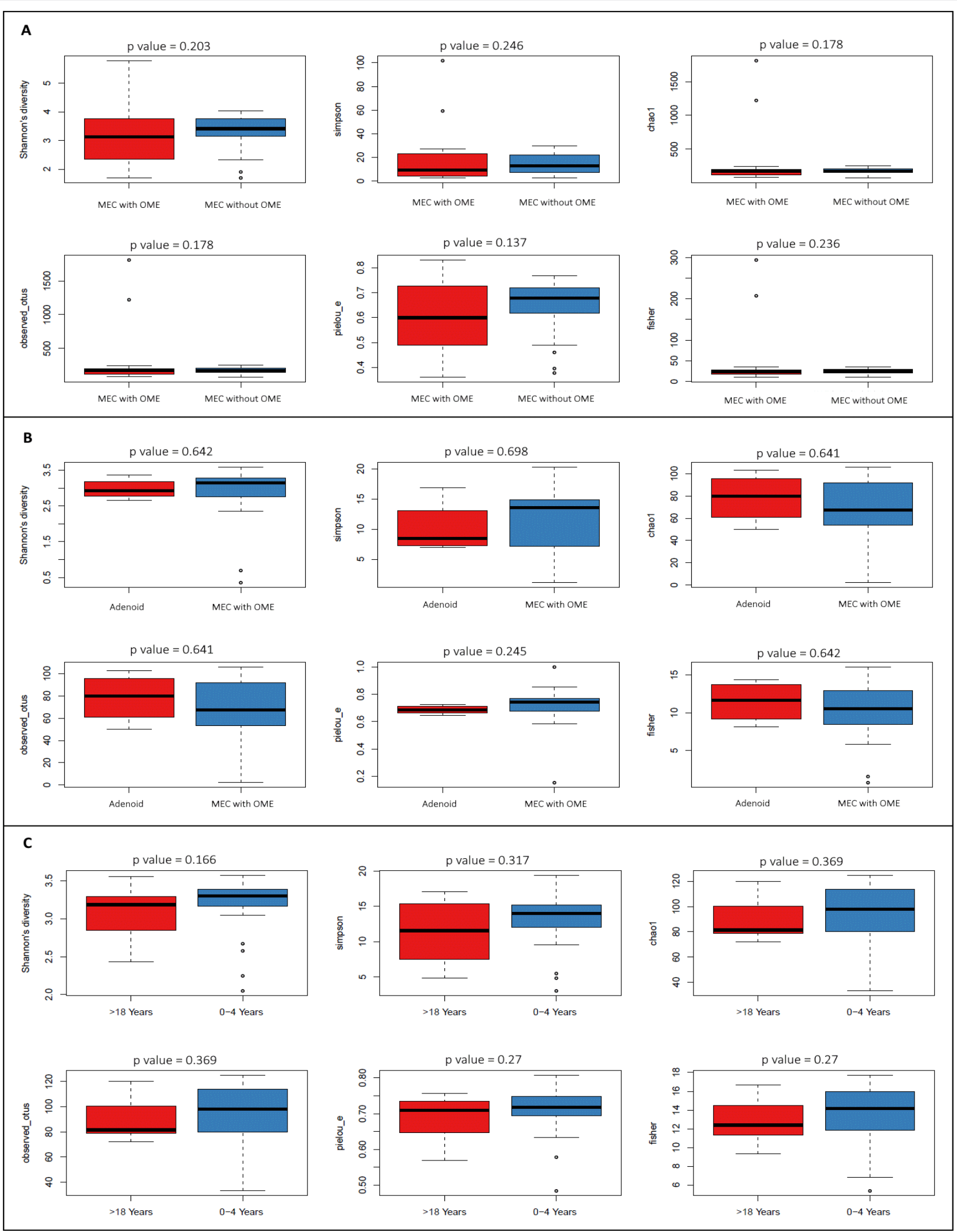

Supplementary Figure 1. a) Alfa diversity analysis of mycobiome detected in middle ear cavity samples of the patients with and without effusion, b) the adenoid and the middle ear cavity samples of the patients with effusion, and c) the middle ear cavity samples of the children and adults. 